\title{
Quantification of Esophageal Angulation in Sigmoid Achalasia: Are We There Yet?
}

TO THE EDITOR: We read the manuscript entitled "Morphologic restoration after peroral endoscopic myotomy in sigmoidtype achalasia" with great interest. ${ }^{1}$ In this manuscript, the authors evaluated the change in esophageal tortuosity after per-oral endoscopic myotomy (POEM) as described by the "Descriptive rules for achalasia of the esophagus." ${ }^{2}$ Timed barium esophagogram (TBE) is commonly utilized to document the success after POEM or any other treatment modality. The traditional rules of assessing the clinical success using TBE, ie, greater than $50 \%$ reduction in barium height may not apply to sigmoid achalasia. ${ }^{3}$ Therefore, other parameters such as change in esophageal angulation, reduction in diameter of esophagus, and widening of esophagogastric junction opening are used to objectify the treatment outcomes in these cases.

In this study by Yoon et al, ${ }^{1}$ the mean angle of esophageal tortuosity increased from $91.5 \pm 13.9^{\circ}$ before POEM to 114.6 $\pm 17.5^{\circ}$ after POEM signifying a reduction in the esophageal burden. ${ }^{1}$ Similar conclusions were drawn in another recent study by Maruyama and colleagues, ${ }^{4}$ where the average esophageal angulation changed from $88.4 \pm 23.1^{\circ}$ before POEM to $109.5 \pm 16.7^{\circ}$ after POEM $(P<0.01)$. The results of both these studies suggest that there is no difference in the short-term outcomes between sigmoid achalasia $\left(90-135^{\circ}\right)$ and advanced sigmoid achalasia $(<$ $\left.90^{\circ}\right) .{ }^{1,4}$ Besides these studies, a couple of other studies with relatively longer follow-up concluded that the pre-operative degree of esophageal flexion does not affect the outcomes of surgery in sigmoid achalasia. ${ }^{5,6}$ The outcomes in these studies compels us to question the utility of quantifying the esophageal angulation or change in esophageal angulation if there is no effect on the final consequences.

We evaluated and compared serial TBE in 2 cases with sigmoid achalasia who underwent POEM at our center. The first case was classified as sigmoid achalasia with an angulation of $113^{\circ}$ before
POEM (Fig. 1A). Post-POEM barium esophagogram revealed an increase of $12^{\circ}$ in the flexion angle at 6 months in concordance with a significant improvement in symptoms (Eckardt 6 to 1) (Fig. 1B). Barium esophagogram done at 2 years revealed a substantial reduction in the flexion angle from $125^{\circ}$ to $109^{\circ}$ signifying an increase in the esophageal burden (Fig. 1C). However, no worsening was noticed in the symptom scores.

In the second case, there could be several possible angulations and we were not sure which one represented the most valid angle (Fig. 2A). There was an increase in the flexion angle on TBE done on day-2 of the POEM procedure (Fig. 2B). Although, the improvement in tortuosity was maintained the symptom scores increased significantly (Eckardt 1 to 5) at 1-year follow-up (Fig. 2C).

The findings in our cases suggest that "one size does not fit all" and follow-up studies are required before considering the "angles" as the surrogate markers for esophageal burden.

In addition, the technique of gauging esophageal flexion deformity needs to be validated and standardized in quality studies. We acknowledge the fact that firm conclusions cannot be drawn based on the results of just 2 cases that we described in this report. In this study by Yoon and colleagues, ${ }^{1}$ the diameter of esophagus and esophagogastric junction opening to assess objective changes in TBE after POEM have also been described. In fact, these parameters are easier to quantify when compared to fine changes in esophageal angles which may be confounded by interobserver variability.

\section{Zaheer Nabi* and D Nageshwar Reddy Asian institute of Gastroenterology, Hyderabad, India}

1. Yoon HJ, Lee JE, Jung DH, et al. Morphologic restoration after peroral endoscopic myotomy in sigmoid-type achalasia. J Neurogastroenterol Motil 2020;26:67-73. 

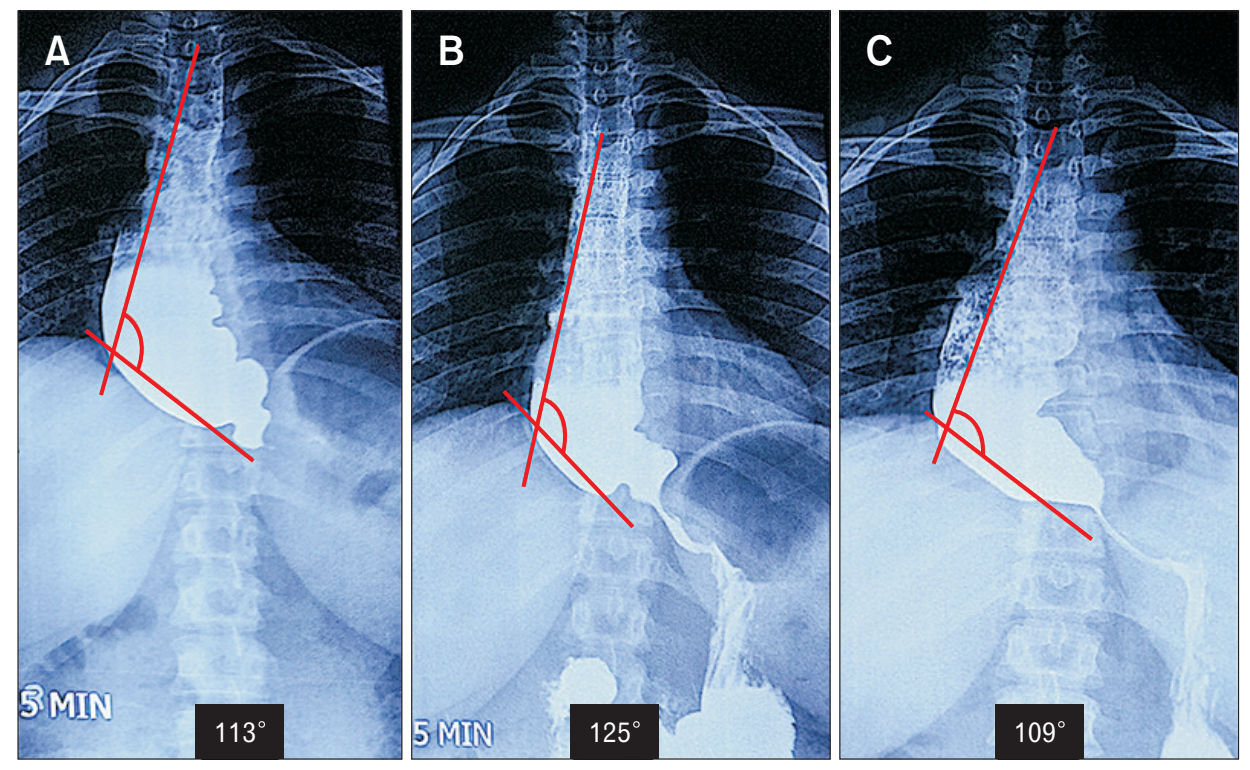

Figure 1. Serial timed barium esophagogram in a case with sigmoid achalasia. (A) Pre-per-oral endoscopic myotomy (pre-POEM) esophagogram revealing significant stasis and angulation of $113^{\circ}$. (B) Post-POEM esophagogram at 6 months revealing free flow of barium with slight change in angulation $\left(125^{\circ}\right)$. (C) Post-POEM esophagogram at 2 -years revealing a reduction in angle $\left(109^{\circ}\right)$.
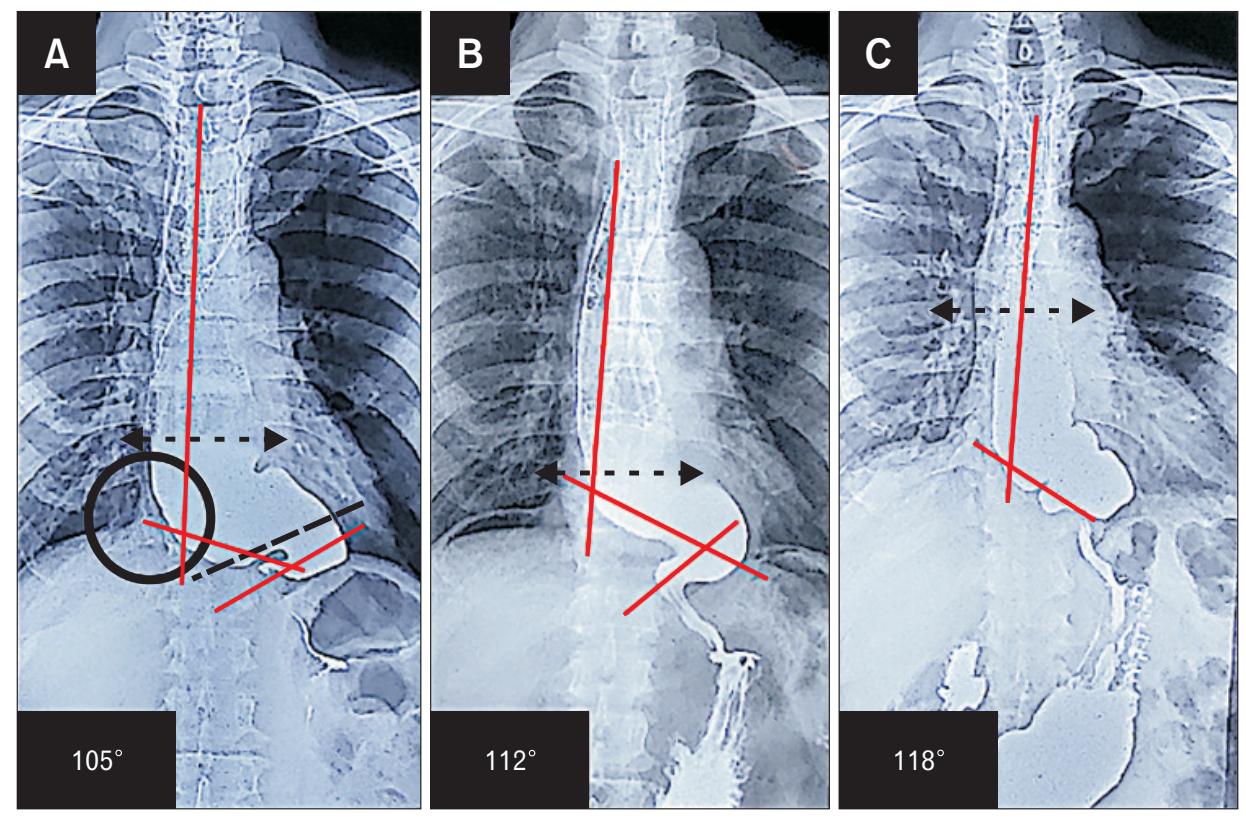

Figure 2. Serial films of timed barium esophagogram in a case with advanced sigmoid esophagus. (A) Pre-per-oral endoscopic myotomy (pre-POEM) esophagogram revealing significant flexion deformity of lower esophagus (black circle). (B) Post-POEM esophagogram revealing a significant reduction in esophageal tortuosity and free-flow of barium across gastroesophageal junction. (C) Post-POEM esophagogram at 1 year shows the preservation of initial improvement in esophageal tortuosity (note the increase in barium column height: 2 sided dashed arrows).

2. Japan Esophageal Society. Descriptive rules for achalasia of the esophagus, June 2012: 4th edition. Esophagus 2017;14:275-289.

3. American Society for Gastrointestinal Endoscopy PIVI Committee; Chandrasekhara V, Desilets D, Falk GW, et al. The American Society for Gastrointestinal Endoscopy PIVI (Preservation and Incorporation of Valuable Endoscopic Innovations) on peroral endoscopic myotomy. Gastrointest Endosc 2015;81:1087-100, e1.

4. Maruyama S, Taniyama Y, Sakurai T, et al. Per-oral endoscopic myotomy (POEM) for a sigmoid type of achalasia: short-term outcomes and changes in the esophageal angle. Surg Endosc Published Online First: 11 Oct 2019. doi: 10.1007/s00464-019-07180-4.
5. Tsuboi K, Omura N, Yano F, et al. Impact of esophageal flexion level on the surgical outcome in patients with sigmoid esophageal achalasia. Surg Today 2017;47:1339-1346.

6. Kato R, Nakajima K, Takahashi T, et al. Validation of new Japanese classification system for esophageal achalasia. Esophagus 2019;16:252-257.

\section{Financial support: None.}

Conflicts of interest: None.

Author contributions: Zaheer Nabi was involved in collecting and interpreting data and drafting the manuscript; D Nageshwar Reddy was involved in providing critical inputs to the manuscript; and both the authors approved the final version of the manuscript. 